\title{
CODING-GAIN-BASED COMPLEXITY CONTROL FOR H.264 VIDEO ENCODER
}

\author{
Ming-Chen Chien ${ }^{1,2}$, Zong-Yi Chen ${ }^{I}$, and Pao-Chi Chang ${ }^{l}$ \\ 1 Department of Communication Engineering, National Central University, Taiwan \\ 2 Department of Electrical Engineering, Chin Min Institute of Technology, Taiwan
}

\begin{abstract}
The allowable computational complexity of video encoding is limited in a power-constrained system. Different video frames are associated with different motions and contexts, and so are associated with different computational complexities if no complexity control is utilized. Variation in computational complexity leads to encoding delay jittering. Typically motion estimation (ME) consumes much more computational complexity than other encoding tools. This work proposes a practical complexity control method based on the complexity analysis of an H.264 video encoder to determine the coding gain of each encoding tool in the video encoder. Experiments performed on a programming optimized source code show that the computational complexity associated with each frame is well controlled below a given limit with very little R-D performance degradation under a reasonable constraint comparing to the unconstrained case.
\end{abstract}

Index Terms - Complexity control, complexity allocation, video encoder, H.264

\section{INTRODUCTION}

The real-time video encoding is an important element for many applications over various wireless networks. To avoid encoding delay jittering, the available encoding time of each video frame, $T_{F C}$, is limited in the real-time video encoding system and can be defined as

$$
T_{F C}=\frac{1}{f r}
$$

where $f r$ represents the frame rate. The limited encoding time of each frame limits the available computational complexity of each frame, $C_{F C}$, which can be defined as

$$
C_{F C}=C_{P R C} \cdot T_{F C}=\frac{C_{P R C}}{f r}
$$

where $C_{P R C}$ represents the clock rate of the processor. However, the $C_{P R C}$ of the processor embedded in wireless handsets is limited and hence $C_{F C}$ is also limited.

Optimal complexity control aims to control the encoding complexity of each frame under a given limit while achieving optimal R-D performance as follows:

$$
\begin{aligned}
& \min J=\min \{D+\lambda R\} \\
& \text { s.t. } \\
& c_{F} \leq C_{F C}
\end{aligned}
$$

where $D$ denotes distortion; $R$ denotes bit rate; $\lambda$ denotes the Lagrange multiplier; $J$ denotes the $R-D$ cost, and $c_{F}$ denotes the complexity used for a frame.

Traditionally, the complexity constraint is computed in the frame layer as described above. For typical MPEG-like video encoders, a frame is partitioned into a number of MBs while an $\mathrm{MB}$ is the basic encoding unit. Different MBs have various motions and contexts and hence are associated with different complexities. Therefore, the allocation of $C_{F C}$ among MBs is a critical problem. Typically, MPEG-like video encoders use many encoding tools, such as ME, DCT, Q, entropy coding and others. Different encoding tools may exhibit substantially different coding efficiency. Accordingly, allocating complexity among encoding tools is another key problem.

A metric of coding gain which represents the coding efficiency has been proposed [4] as follows:

$$
\begin{aligned}
& C G=\Delta J / \Delta C \\
& \Delta J=\Delta D+\lambda \Delta R
\end{aligned}
$$

where $\Delta C$ represents the increase in complexity when an encoding tool is adopted; $\Delta D$ represents the decrease in distortion; $\Delta R$ represents the decrease in rate, and $\lambda$ is the Lagrange multiplier. However, a proper $\lambda$ is not easily determined. When the rate control is turned on for a target rate, $\Delta R$ becomes nearly zero, and $\Delta J$ equals $\Delta D$ :

$$
C G=\Delta D / \Delta C
$$

A few works of complexity control have been conducted [2],[3],[4],[5]. The optimization formula of the first C-R-D model [2] is too complicated to be solved in closed form. Also, an MHM-based method for allocating complexity for ME among MBs, which was not optimal, was also proposed in that study. A statistical optimal operation mode for a sequence in a complexity-constrained video encoding system has also been proposed [3]. However, an optimal operation mode could be optimal for a frame but inadequate for another frame. A complexity allocation method for $\mathrm{ME}$ based on the costcomplexity curve has been proposed [4]. A C-R-D optimization for H.264 ME has also been proposed [5]. It proposed two Lagrange multipliers to terminate the complexity-inefficient ME rounds and thus increase coding efficiency. Typically ME consumes most complexity with a large variation between MBs. In general, optimal complexity control algorithms are difficult 
to apply to practical real-time video encoders because of their large computational overhead. To the best of our knowledge, no practical complexity control that is efficiently enough and operates in real time exists for an H.264 video encoder.

Based on complexity analysis of a programming optimized H.264 code, X264 [10], this work proposes a simple and practical complexity control method which can control the encoding complexity of each frame under a given limit while achieving very good R-D performance.

This paper is organized as follows. Section 2 proposes a practical complexity control method based on the results of complexity analysis. Section 3 presents experimental results, and section 4 draws conclusions.

\section{PROPOSED COMPLEXITY CONTROL}

For a typical MPEG-like video encoder, Figure 1 displays the encoding block diagram of an MB. DCT, Q, $Q^{-1}$, IDCT have been collectively denoted by PRECODING [2]. This paper follows this notation, and divides the encoder into three major encoding tools - ME, PRECODING, and entropy coding.

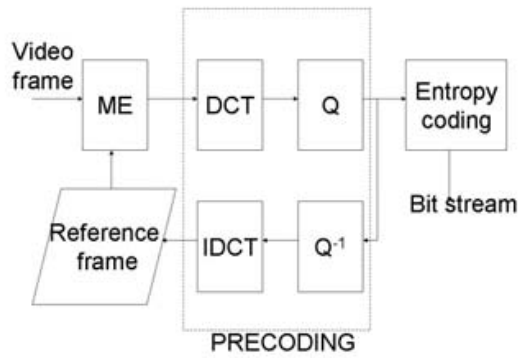

Fig. 1 Basic block diagram of a video encoder

Highly efficient complexity control should be performed by allocating complexity to the encoding tools with higher coding gain. This work conducts experiments with the options presented in Table I to analyze the coding gains of various encoding tools in the modern H.264 encoder. The metric of coding gain is given by (6), where $\Delta D$ is represented by $\triangle P S N R$, which represents the increase in PSNR, and $\triangle C$ is measured by the number of CPU clocks spent on a piece of code. Table II presents the results, which will be discussed in the following subsection.

Table I.

Options for complexity analysis

\begin{tabular}{|l|l|}
\hline Video source & $\begin{array}{l}\text { Foreman QCIF, Carphone } \\
\text { QCIF }\end{array}$ \\
\hline Fast ME & Diamond \\
\hline Target rate & $103 \mathrm{k}$ bps \\
\hline Frame rate & 20 \\
\hline Number of reference frames & 1 \\
\hline GOP type & IPPPP \\
\hline CPU & Intel Pentium 4 2.66G Hz \\
\hline RAM & $512 \mathrm{M}$ bytes \\
\hline MMX tech. & On for SAD computation \\
\hline Source code of H.264 & X264 \\
\hline
\end{tabular}

Table II.

Coding gain of each encoding tool

\begin{tabular}{|l|l|}
\hline Encoding tool & $\begin{array}{l}\text { Coding gain } \\
(\mathrm{db} / \mathrm{kclks})\end{array}$ \\
\hline CABAC (compare to CAVLC) & $9.17 \mathrm{e}-4$ \\
\hline half pixel ME & $2.88 \mathrm{e}-3$ \\
\hline Deblocking filter & $8.54 \mathrm{e}-4$ \\
\hline Quarter pixel ME & $4.45 \mathrm{e}-4$ \\
\hline $8 \times 8$ partition mode & $1.42 \mathrm{e}-4$ \\
\hline $16 \times 8$ \& 8x16 partition mode & $4.63 \mathrm{e}-5$ \\
\hline Sub8x8 partition mode & $4.7 \mathrm{e}-5$ \\
\hline $4 \times 4$ Intra & $5.22 \mathrm{e}-6$ \\
\hline 5 reference frames & $4.06 \mathrm{e}-5$ \\
\hline
\end{tabular}

\subsection{Complexity Allocation}

The complexity allocation allocates complexity from frame layer to MB layer. It should be performed before the first MB in a frame is encoded. When the video encoder starts to encode a frame, it should do some initialization before encoding slices. Complexity control records the complexity consumed by the initialization, which is denoted by $C_{\text {Finit }}$. The complexity budget of encoding all slices in a frame is $C_{S L s}$. After the slices are encoded, deblocking filtering can be performed; it is followed by updating references and other necessary tasks. The complexity of these tasks after the encoding of slices, $C_{\text {Fother }}$, should be reserved. The deblocking filter is suggested to be adopted because it has high coding gain as shown in Table II and proposed elsewhere [6]. $C_{F o t h e r}$ is smaller than $C_{S L s}$ as displayed in Fig. 2, and it does not vary greatly. It can be regarded as a constant and can be estimated from the previous frame. Accordingly, before the slices are encoded, by measuring $C_{\text {Finit }}$ and reserving $C_{\text {Fother }}, C_{S L s}$ can be allocated by
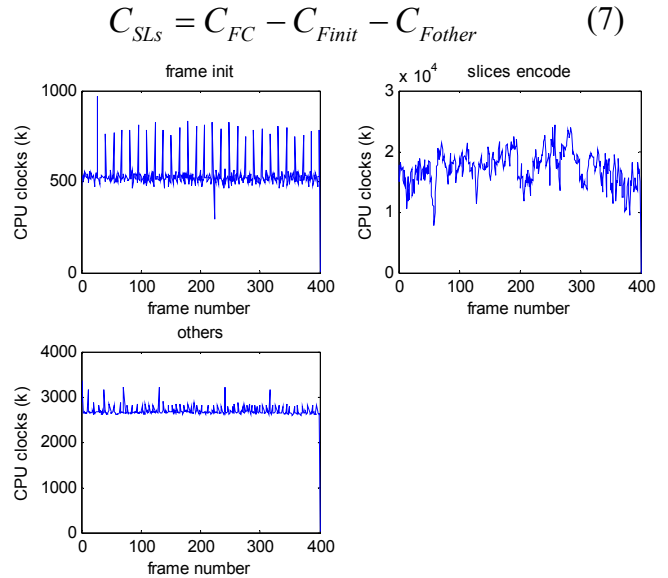

Fig. 2 Complexity consumption in the frame layer

The operation of the slice layer is very simple. Only a short slice header is added. The complexity of encoding all slice headers in a frame is small and can be treated as a constant. It is denoted by $C_{S L h s}$. Therefore, the complexity of encoding all MBs in a frame, $C_{M B s}$, can be allocated according to

$$
C_{M B s}=C_{S L s}-C_{S L h s}
$$


Each MB can adopt ME, PRECODING and entropy coding. Typically, ME consumes most of the complexity, as shown in Fig. 3. It is the main object on which complexity control will be performed. The modern entropy coding tool CABAC has a high coding gain, as shown in Table II and elsewhere [6]. Its adoption is recommended. The modern video encoding standard H.264 significantly simplifies DCT operation [6]. Hence, PRECODING has high coding gain, and is destined to be adopted. Some early termination algorithms for PRECODING have been proposed to skip the PRECODING for the MB with small residual signals [11]. All such algorithms with high efficiency can be utilized. As described above, the complexity for PRECODING and entropy coding should be reserved. The complexity budget $C_{M E s}$ can be allocated using

$$
C_{\text {MEs }}=C_{M B s}-C_{\text {MBother }} \times M
$$

where $C_{M B o t h e r}$ denotes the complexity reserved for PRECODING and entropy coding of a $\mathrm{MB}$ and $M$ is the number of MBs in a frame. Figure 3 shows $C_{M B o t h e r}$ is relatively small and its variation is much smaller than $C_{M E}$, the complexity for ME of a MB. Therefore, $C_{M B o t h e r}$ can be treated as a constant and can be estimated statistically by running test video sequences in advance. The complexity compensation described below will eliminate the estimation error.
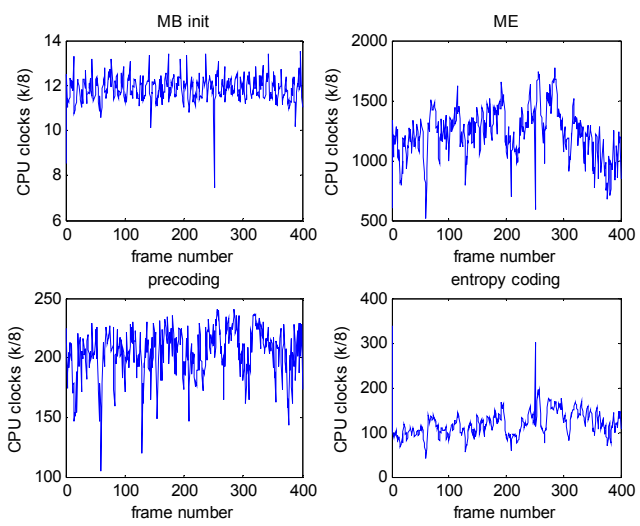

Fig. 3 Complexity consumption in the MB layer

The complexity allocation for $\mathrm{ME}$ among $\mathrm{MBs}$ is suggested to be weighted by $\operatorname{COSTO}$ as

$$
C_{M E}(i)=C_{M E s} \times \frac{\operatorname{COST}_{i}}{\sum_{j=1}^{M} \operatorname{COST} 0_{j}}, \quad i=1,2, \ldots, M
$$

where COST0 represents the cost of $\mathrm{ME}$ with zero $\mathrm{MV}$ in $16 \times 16$ partition mode. This equation is simple but meaningful because COSTO contains information about context and motion. Since the MB with larger motion or more complex context has larger COST0, it deserves larger complexity budget. Otherwise, a larger bit rate and larger distortion will be generated.

\subsection{ME Flow in Decreasing order of CG}

According to the coding gain in Table II, the ME flow in Fig. 4 is suggested. The resulting operation order is similar to that suggested elsewhere [5] but the adoption of $4 \times 4$ Intra prediction is different. Table II reveals that the coding gain of $4 \times 4$ Intra for inter frames is very low, because most MBs in the inter frame choose inter mode as the best mode. However, 4x4 Intra prediction is beneficial to MBs that choose the Intra mode. The tendency to Intra mode is examined by comparing $16 \times 16 \mathrm{ME}$ and $16 \times 16$ Intra prediction. If the 16x16 Intra prediction yields a better performance, $4 \times 4$ Intra prediction can be utilized to reduce the residual signal. Otherwise, $4 \times 4$ Intra prediction is not used.

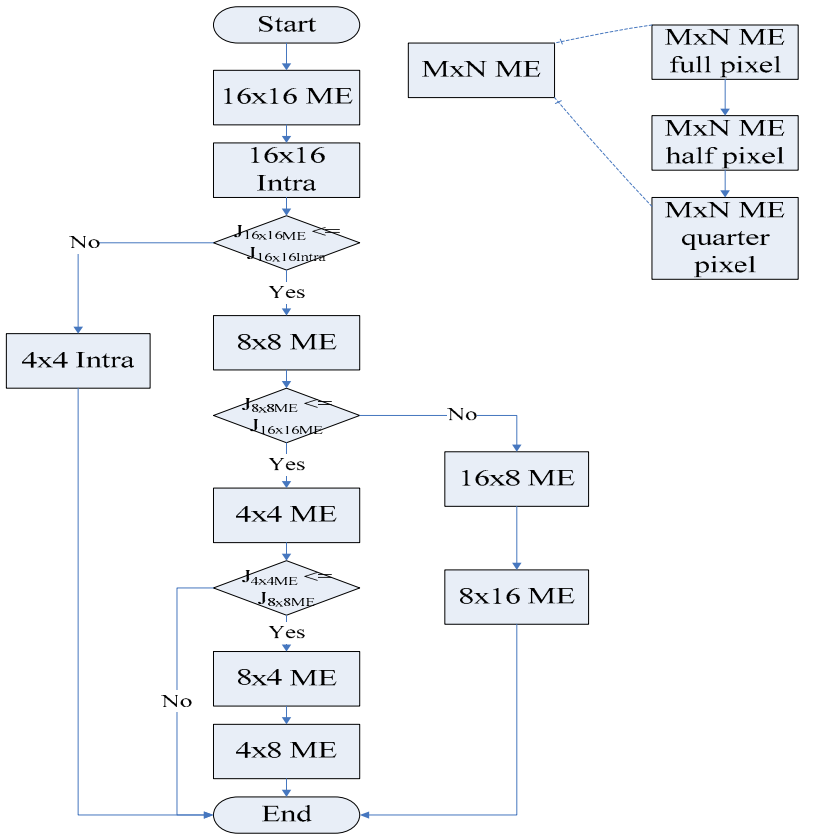

Fig. $4 \mathrm{ME}$ flow in decreasing $\mathrm{CG}$ of encoding tools

\subsection{Complexity Check and Compensation}

After each computation of SAD and the R-D cost, the used complexity $C_{M E u s e d}$ is examined. If $C_{M E u s e d}$ exceeds $C_{M E}$, the ME process terminates. Otherwise, the ME process continues.

Any efficient early termination algorithm for PRECODING can be employed. Complexity compensation described below will distribute the saved complexity.

After the whole process of the MB encoding is complete, the balance $C_{\text {MBbalance }}$ between the used complexity $C_{M B u s e d}$ and the budget $C_{M B}$ is given by

$$
C_{\text {MBbalance }}=C_{M B}-C_{M B u s e d}
$$

where $C_{M B}$ is obtained by

$$
C_{M B}=C_{M E}+C_{M B o t h e r}
$$

Then $C_{M B b a l a n c e}$ is distributed uniformly to the remaining MBs in that frame.

\section{EXPERIMENTAL RESULTS}

The options of experiments for the proposed practical complexity control are shown in Table III. The complexity metric is the number of CPU clocks used by an encoding tool, as measured by the 'rdtsc' instruction of an Intel CPU [7].

Figure 5 indicates that the complexity is well controlled under the given limit. The complexity of each frame rarely exceeds the bound. Figure 6 and 7 show that the rate and PSNR 
under complexity control are both very close to those in the unconstrained case. Figure 8 plots the R-D performance with Foreman video sequence under various complexity constraints, where $\mathrm{Cfm}$ denotes the maximum complexity of a frame without complexity constraint. When $C_{F C}$ is down to $72 \%$ of Cfm, the PSNR obtained by this algorithm only degrades less than $0.5 \mathrm{~dB}$ at the same rate. When $C_{F C}$ is down to $58 \%$ of $\mathrm{Cfm}$, the PSNR obtained by this algorithm degrades no more than 1 $\mathrm{dB}$ at the same rate. Experiments with another video source 'Carphone' yield similar results.

Table III.

Options for complexity control

\begin{tabular}{|l|l|l|l|l|l|}
\hline $\begin{array}{l}C_{F \max } \\
(\mathrm{clk})\end{array}$ & source & QP & $\begin{array}{l}\text { Rate } \\
\text { control }\end{array}$ & Fast ME & $\begin{array}{l}\text { Complexity } \\
\text { metric }\end{array}$ \\
\hline $23 \mathrm{M}$ & Foreman & 29 & off & Diamond & CPU clock \\
\hline
\end{tabular}

\section{CONCLUSION AND FUTURE WORK}

This work proposes an efficient complexity control method with very little degradation of R-D performance. The proposed method, which has very low overhead, is also very practical.

\section{REFERENCES}

[1] "Draft ITU-T recommendation and final draft international standard of joint video specification (ITU-T rec. H.264/ISO/IEC 14496-10 AVC)", in JVT of ISO/IEC MPEG and ITU-T VCEG, JVT-G050, 2003.

[2] Z. He and Y. F. Liang, "Power-Rate-Distortion analysis for wireless video communication under energy constraints," IEEE Trans. Circuits Syst. Video Technol., vol. 15, no. 5, pp. 645-658, May 2005.

[3] D. N. Kwon and P. F. Driessen, "Performance and computational optimization in configurable hybrid video system," IEEE Trans. Circuits Syst. Video Technol., vol. 16, no. 1, pp. 3142, Jan. 2006.

[4] C. Kim and J. Xin, "Hierarchical complexity control of motion estimation for H.264/AVC," MITSUBISHI ELECTRIC RESEARCH LABORATORIES, TR2006-004, Dec. 2006. Available: http://www.merl.com

[5] Y. Hu, Q. Li, S. Ma, and C. C. J. Kuo, "Joint rate-distortioncomplexity optimization for H.264 motion search," in Proc. ICME 2006, pp. 1949-1952.

[6] E. G. Richardson, H.264 and MPEG-4 Video Compression. John Wiley \& Sons, 2003.

[7] http://www.intel.com

[8] J. Ostermann, J. Bormans, P. List, D. Marpe, M. Narroschke, F. Pereira, T. Stockhammer, and T. Wedi, "Video coding with H.264/AVC: Tools, performance, and complexity," IEEE Circuits Syst. Mag., vol. 4, no. 1, pp. 7-28, Apr. 2004.

[9] Joint Model reference software version 10, Available: http://iphome.hhi.de/suehring/tml/index.htm

[10] x264, Available: http://developers.videolan.org/x264.html.

[11] Z. Chen, P. Zhou, and Y. He, "Fast Integer Pel and Fractional Pel Motion estimation in for JVT," JVT-F017r1.doc, Joint Video Team (JVT) of ISO/IEC MPEG \& ITU-T VCEG, $6^{\text {th }}$ meeting, Awaji, Island, JP, 5-13 Dec. 2002.

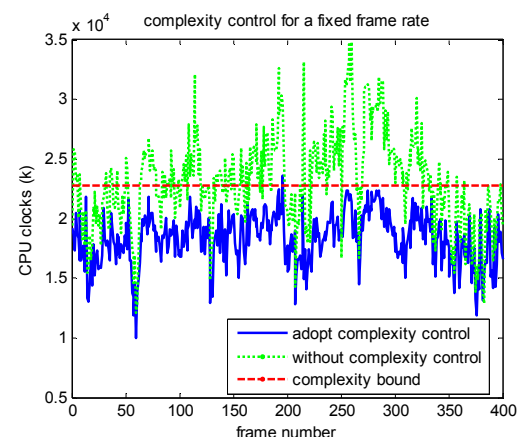

Fig. 5 Comparisons of computational complexity with and without complexity control

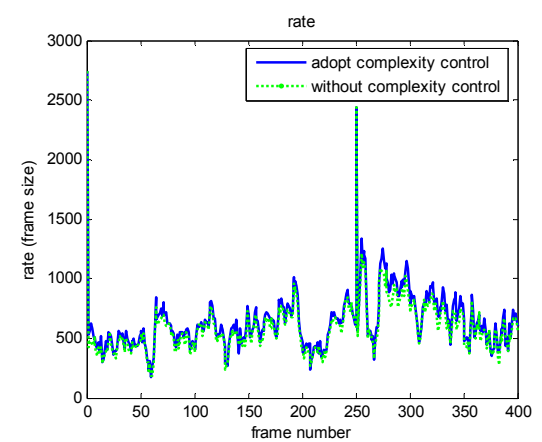

Fig. 6 Comparisons of rate with and without complexity control

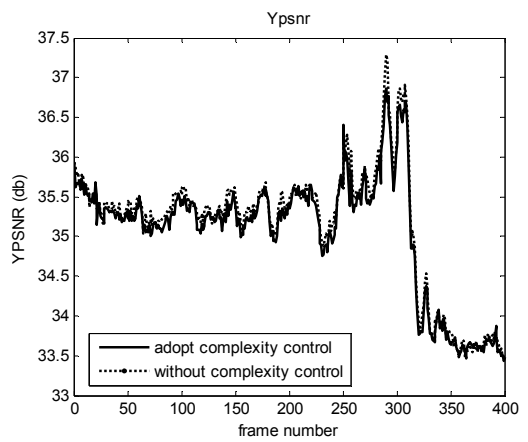

Fig. 7 Comparisons of YPSNR with and without complexity control

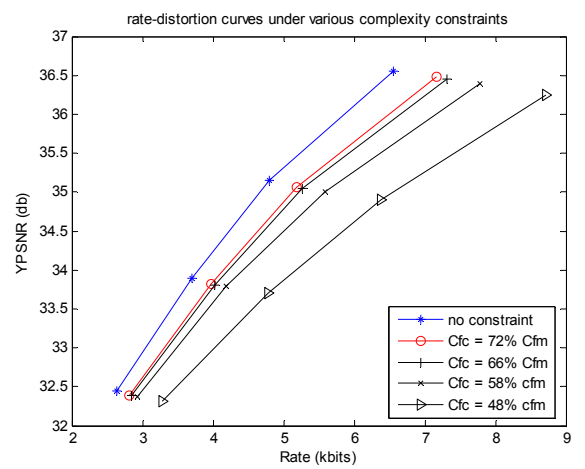

Fig. 8 R-D performance under various complexity constraints 